MARCin Malawski (Warszawa)

\title{
VALUES OF MAJORITY VOTING GAMES WITH DISTRUST OPERATORS
}

Abstract. A distrust operator, describing a kind of agreement among a group of players, transforms any characteristic function game to another game. In this new game, a player from this group can legally access a coalition if and only if all players from the group do the same. A formula for the Shapley value of games obtained by applying distrust operators to one man-one vote majority voting games is given, and the cases in which such an "agreement" is profitable to its parties are discussed. We also prove two theorems concerning the limit behaviour of values of voting games with distrust operators when the number of players tends to infinity but the winning majority percentage remains constant.

1. Introduction. Games and operators. We study the values of majority voting games transformed by distrust operators. A distrust operator, first introduced in [2] and closely related to representation and association agreements studied in [1] and [3], is an example of an additional structure on the set of players which transforms every cooperative game to another cooperative game. An interesting question is whether forming such a structure is profitable (in some sense) for players who form it. We give a partial answer to this question in the case where the game is a one man-one vote majority voting game and profitability is understood as increasing the Shapley values of the players involved.

Having introduced the necessary notions in the first two sections, we give an explicit formula for the Shapley values of majority voting games with distrust operators in Section 3. In Section 4 we present examples of both profitable and unprofitable operators together with some general statements on profitability. The last section describes the limit behaviour of values of

2000 Mathematics Subject Classification: 91A12, 91A06.

Key words and phrases: majority voting games, Shapley value, distrust operator.

This work has been supported by the KBN research grant 1 H02B 01515 . 
"large" voting games with distrust operators when the number of players tends to infinity and the minimal winning majority required is a constant fraction of the number of players. Two cases are discussed there: in the first one, the number of players forming an agreement remains constant, and in the second one it is a constant fraction of the number of all players.

Let $N=\{1, \ldots, n\}$ be a finite set of players. Every subset of $N$ is called a coalition. An $n$-person cooperative game (characteristic function game) is the pair $(N, v)$, where $v$ is the characteristic function - any real function defined on the set $\mathcal{N}$ of all coalitions and satisfying $v(\emptyset)=0$. It is a standard practice to identify every cooperative game with its characteristic function. An important subclass of cooperative games are one man-one vote majority voting games with the characteristic functions defined by

$$
v_{n}^{\mu}(S)= \begin{cases}1 & \text { if } \# S \geq \mu \\ 0 & \text { if } \# S<\mu .\end{cases}
$$

Here, $n$ is the number of players, \#S denotes the cardinality of the coalition $S(\# S \leq n)$, and $\mu$ is the minimum winning majority (quorum) - any number such that $n / 2<\mu \leq n$. Without loss of generality we can assume that $\mu$ is an integer.

Majority voting games, on the other hand, belong to an important subclass of characteristic function games - simple games. A cooperative game $(N, w)$ is a simple game if the following conditions hold:

1. for every coalition $S \subset N, w(S)=0$ or $w(S)=1$,

2. $w(N)=1$,

3. if $S \subset T$, then $w(S) \leq w(T)$ (monotonicity).

In a simple game $w$, winning coalitions are those belonging to $w^{-1}(1)$, and losing coalitions are those in $w^{-1}(0)$. Player $i$ is decisive in a coalition $S$ in a simple game $w$ if $w(S)=1$ and $w(S \backslash\{i\})=0$, i.e. if $i$ 's leaving the winning coalition $S$ changes it to a losing coalition. (In majority voting games, the exit of $i$ causes $S$ to lose majority.)

Denote by $\mathcal{G}_{n}$ the set of all $n$-person cooperative games, and by $\mathcal{G}^{*}=$ $\bigcup_{n=1}^{\infty} \mathcal{G}_{n}$ the set of all cooperative games with finite numbers of players. For any set $N$ of players and any coalition $Q \subset N$ with $\# Q \geq 2$, the distrust operator $D_{Q}: \mathcal{G}_{n} \rightarrow \mathcal{G}_{n}$ assigns to any game $v \in \mathcal{G}_{n}$ a new game $d=D_{Q}(v) \in \mathcal{G}_{n}$ defined by

$$
d(S)= \begin{cases}v(S) & \text { if } Q \subset S, \\ v(S \backslash Q) & \text { if } Q \backslash S \neq \emptyset .\end{cases}
$$

A distrust operator $D_{Q}$ can be interpreted as a pre-play agreement signed by all players belonging to $Q$, stating that any player from the set $Q$ may join any coalition $T$ if and only if all players in $Q$ do the same: an access to $T$ may only be collective ("all or none"). Even though the term "agreement" 
is established for similar operators [1], we prefer to use the term "operator" instead in order to avoid disturbing "distrust agreements". However, such an arrangement between players may well make sense; a group of players can agree upon it, for instance, expecting to strengthen their bargaining position, and we shall see later that indeed this is often the case.

It is straightforward to check that for every $N \supset Q, D_{Q}$ is a linear projection of the set $\mathcal{G}_{n}$ to $\mathcal{G}_{n}$ and that it transforms simple games to simple games. However, if $v$ is a voting game, then $D_{Q}(v)$ need not be a voting game. If, for example, $Q=\{1,2,3\}$, then in the game $D_{Q}\left(v_{7}^{4}\right)$ five-person coalitions containing exactly two of players 1,2 and 3 are losing, but fourperson coalitions $\{1,2,3, k\}(k>3)$ are winning.

2. Values. Values are tools for measuring the "power" of players in cooperative games. A value is any vector-valued function $\psi$ defined on $\mathcal{G}^{*}$ such that for every $n, \psi\left(\mathcal{G}_{n}\right) \in \mathbb{R}^{n}$. The components of $\psi(v)$, denoted by $\psi_{k}(v)$, are called individual values of players and should somehow reflect the players' positions in the game $v$.

The by far best-known and most commonly accepted value is the Shapley value $\phi$, first introduced in [6] and given by the formula

$$
\phi_{i}(v)=\sum_{T \subset N} \frac{(t-1) !(n-t) !}{n !}(v(T)-v(T \backslash\{i\})
$$

where $t=\# T$.

When $v$ is a simple game, it is obvious that the difference $v(T)-v(T \backslash\{i\})$ is always equal to 0 or 1 , and it equals 1 if and only if player $i$ is decisive in the coalition $T$. Thus, for simple games - and in particular for voting games - the Shapley value can be expressed in a simpler form

$$
\phi_{i}(v)=\sum_{T \in \Delta(i, v)} \frac{(t-1) !(n-t) !}{n !}
$$

where $t=\# T$ and $\Delta(i, v)$ is the set of all coalitions in which player $i$ is decisive (in the game $v$ ).

While computing the values of particular games, it is often useful to apply the following well-known probabilistic interpretation of the Shapley value (see e.g. [6] or [5]). For a given player $j \in N$ and a permutation $\pi$ of the set $N$ denote by $H_{\pi, j}$ the coalition consisting of player $j$ and of all players who precede $j$ in the order determined by $\pi$ :

$$
H_{\pi, j}=\{k: \pi(k) \leq \pi(j)\} .
$$

Now if we order all players in $N$ at random and assume all orders on (= permutations of) the set $N$ to be equiprobable, then $\phi_{i}(v)$ is the expected value of the contribution of player $i$ to the (random) coalition $H_{\pi, i}$, i.e., of the difference $v\left(H_{\pi, i}\right)-v\left(H_{\pi, i} \backslash\{i\}\right)$. 
In particular, when $w$ is a simple game, then this expected value is just the probability that $i$ is decisive in the coalition $H_{\pi, i}$. Since it is clear that for every permutation $\pi$ there is exactly one player $k$ who is decisive in the coalition $H_{\pi, k}$ (in the game $w$ ), we may denote that player by $\delta(\pi)$ and write

$$
\phi_{i}(w)=\mathrm{P}(\delta(\pi)=i) \quad \text { for } i=1, \ldots, n .
$$

Moreover, the Shapley value is known to have two fundamental properties: efficiency and equal treatment property, which also often help compute values of games and will be used in what follows. Efficiency means that for every $n$-person game $v$ we have $\sum_{i=1}^{n} \phi_{i}(v)=v(N)$ (and thus, in particular, for every $n$-person simple game $\left.w, \sum_{i=1}^{n} \phi_{i}(w)=1\right)$. Equal treatment property means that if two players $i, j$ are interchangeable in the game $v$, i.e., if for every coalition $T$ including neither $i$ nor $j$ we have $v(T \cup\{i\})=v(T \cup\{j\})$, then $\phi_{i}(v)=\phi_{j}(v)$.

3. Formulae for the values. We now give explicit formulae for the values of majority voting games before and after applying a distrust operator.

The computation of $\phi\left(v_{n}^{\mu}\right)$ is trivial thanks to efficiency and equal treatment property of the Shapley value; these two properties together immediately imply that

$$
\phi_{i}\left(v_{n}^{\mu}\right)=1 / n \quad \text { for } i=1, \ldots, n .
$$

Now take any coalition $Q \subset N$ and denote its cardinality by $q$. In order to compute the value of $D_{Q}\left(v_{n}^{\mu}\right)$, set

$$
d_{n}^{\mu}=D_{Q}\left(v_{n}^{\mu}\right), \quad \mathrm{d}_{j}=\phi_{j}\left(d_{n}^{\mu}\right), \quad \mathrm{d}_{Q}=\sum_{j \in Q} \mathrm{~d}_{j} .
$$

(Obviously, both $\mathrm{d}_{j}$ and $\mathrm{d}_{Q}$ depend on $n, \mu$ and $q$, but we shall omit these arguments whenever it causes no confusion.) Notice that in the game $\mathrm{d}_{n}^{\mu}$ all players in $Q$ are interchangeable and all players in $N \backslash Q$ are interchangeable. Therefore efficiency and equal treatment property imply that

$$
\mathrm{d}_{j}= \begin{cases}\frac{\mathrm{d}_{Q}}{q} & \text { for } j \in Q, \\ \frac{1-\mathrm{d}_{Q}}{n-q} & \text { for } j \notin Q,\end{cases}
$$

and so it suffices to compute $\mathrm{d}_{Q}$.

It will prove useful to distinguish between three cases:

Case 1: $q \geq \mu$, i.e., the players entering the agreement themselves form a winning coalition (both in $v_{n}^{\mu}$ and in $d_{n}^{\mu}$ ). In this case it is obvious that all players from $N \backslash Q$ are null players (have zero contribution to every 
coalition), so in this case $\mathrm{d}_{Q}=1$. We therefore exclude this case from all further considerations.

Case 2: $\max (q, n-q)<\mu$-neither $Q$ nor $N \backslash Q$ is a winning coalition in any of the two games. By analogy to [4] we shall call this situation the non-interior case.

Case 3: $q<\mu$ and $n-q \geq \mu$, i.e., the players who do not enter the agreement can form a winning coalition. This is the interior case.

In what follows, let us adopt the notation

$$
U_{k}^{l}=(k+1)(k+2) \ldots l=\frac{l !}{k !}
$$

for any two natural numbers $k, l$ such that $l>k$.

THEOREM 1. For $q<\mu$,

$$
\mathrm{d}_{Q}=\left\{\begin{array}{l}
1-\frac{(n-q) !(\mu-1) !}{n !(\mu-q-1) !}=\frac{U_{n-q}^{n}-U_{\mu-q-1}^{\mu-1}}{U_{n-q}^{n}} \\
\text { if } q>n-\mu(\text { the non-interior case }), \\
\frac{(n-q) !}{n !}\left[\frac{(\mu+q-1) !}{(\mu-1) !}-\frac{(\mu-1) !}{(\mu-q-1) !}\right]=\frac{U_{\mu-1}^{\mu+q-1}-U_{\mu-q-1}^{\mu-1}}{U_{n-q}^{n}} \\
\text { if } q \leq n-\mu(\text { the interior case }) .
\end{array}\right.
$$

Proof. In view of the aforementioned probabilistic interpretation of the Shapley value, for every coalition $T, \mathrm{~d}_{T}$ is the probability that (given that all permutations of $N$ are equiprobable) for a random permutation $\pi$ the player $\delta(\pi)$ belongs to $T$.

Obviously, $i=\delta(\pi)$ if and only if the accession of player $i$ to the set of players who precede him- $H_{\pi, i} \backslash\{i\}$-results in forming a new winning coalition. In the game $d_{n}^{\mu}$, this is possible for player $i$ belonging to $Q$ only if $H_{\pi, i} \supset Q$, i.e., $i$ is the "last" player from the set $Q$. (This follows directly from the definition of $D_{Q}$ : the contributions of players from $Q$ to any coalition which does not contain $Q$ are zero.)

Thus, player $\delta(\pi)$ belongs to $Q$ if and only if the permutation $\pi$ satisfies the following two conditions:

$$
\begin{aligned}
& \pi^{-1}(\{\min (\mu+q, n+1), \ldots, n-1, n\}) \cap Q=\emptyset, \\
& \pi^{-1}(\{\mu, \mu+1, \ldots, n\}) \cap Q \neq \emptyset,
\end{aligned}
$$

i.e., all players from $Q$ are placed at positions $1,2, \ldots, \min (\mu+q-1, n)$, and at least one of them is placed at position $\mu, \mu+1, \ldots$ or $n$.

Denoting the event (1) by $E$ and the event (2) by $F$, we have

$$
\mathrm{d}_{Q}=\mathrm{P}(E \cap F) .
$$


But $\Omega \backslash F \subset E$ : if there are no players from $Q$ at places $\mu, \mu+1, \ldots, n$, then there are no players from $Q$ at places $\mu+q, \mu+q+1, \ldots, n$. Thus $\mathrm{P}(E \cap F)=$ $\mathrm{P}(E)-\mathrm{P}(\Omega \backslash F)$. These probabilities are given by

$$
\begin{aligned}
P(E) & =1 \quad \text { if } q>n-\mu, \\
P(E) & =\left(\begin{array}{c}
\mu+q-1 \\
q
\end{array}\right) /\left(\begin{array}{l}
n \\
q
\end{array}\right)=\frac{(\mu+q-1) !(n-q) !}{(\mu-1) ! n !} \quad \text { if } q \leq n-\mu, \\
P(\backslash F) & =\left(\begin{array}{c}
\mu-1 \\
q
\end{array}\right) /\left(\begin{array}{l}
n \\
q
\end{array}\right)=\frac{(\mu-1) !(n-q) !}{(\mu-q-1) ! n !},
\end{aligned}
$$

which ends the proof.

4. Profitability: examples and basic lemmata. Having the formula for $\mathrm{d}_{Q}$, we now address the question of profitability of distrust operators in terms of the Shapley value. We shall say that $D_{Q}$ is profitable in the game $v$ if the sum of the Shapley values of all players in $Q$ is greater in the game $D_{Q}(v)$ than in $v$; if the reverse inequality holds, $D_{Q}$ is unprofitable. For one man-one vote majority voting games, profitability of $D_{Q}$ in $v_{n}^{\mu}$ is equivalent to the increase of individual Shapley values of all players in $Q$, and thus to $\mathrm{d}_{Q}>q / n$.

In this section and in the next one we shall always assume that $q<\mu$.

EXAMPLE 1 (Unanimity games). In the unanimity game of the grand coalition $(\mu=n)$, regardless of the cardinality of $Q$ the player coming last is always decisive:

$$
\forall \pi \quad \delta(\pi)=\pi^{-1}(n) .
$$

Thus, $\mathrm{d}_{i}=1 / n$ for every $i \leq n$, and $\mathrm{d}_{Q}=q / n$ for every $Q$. The operator $D_{Q}$ is neither profitable nor unprofitable for players in $Q$; we might say that it is neutral in this case.

Lemma 1. In the non-interior case $(q>n-\mu), D_{Q}$ is always profitable unless $\mu=n$.

Proof. We have

$$
\mathrm{d}_{Q}=1-\frac{(n-q) !(\mu-1) !}{n !(\mu-q-1) !}=1-\frac{n-q}{n} \cdot \frac{(\mu-q) \ldots(\mu-1)}{(n-q) \ldots(n-1)}>1-\frac{n-q}{n}
$$

whenever $\mu<n$.

Thus, in the non-interior case, distrust operators are always either neutral (when $v$ is a unanimity game) or profitable (otherwise). This observation allows us to confine all further considerations to the interior case.

EXAmple $2(q=2)$. In this case

$$
\mathrm{d}_{Q}=\frac{\mu(\mu+1)-(\mu-1)(\mu-2)}{n(n-1)}=\frac{2(2 \mu-1)}{n(n-1)}>\frac{2}{n}
$$


(since, as usual, $\mu>n / 2$ ), so two-person "distrust agreements" are strictly profitable.

EXAMPLE $3(q=n-\mu)$. This is the broadest agreement possible in the interior case. We have

$$
\begin{aligned}
\mathrm{d}_{Q} & =\frac{\mu !}{n !}\left[\frac{(n-1) !}{(\mu-1) !}-\frac{(\mu-1) !}{(2 \mu-n-1) !}\right]=\frac{\mu}{n}-\frac{(2 \mu-n) \ldots(\mu-1)}{(\mu+1) \ldots n} \\
& >\frac{\mu}{n}-\frac{2 \mu-n}{n}=\frac{q}{n} .
\end{aligned}
$$

All the above examples suggest that distrust operators are "usually" profitable. However, this is not true. We show below that already threeperson "agreements" are not profitable in many cases, and that in voting games with $\mu=[n / 2]+1$ ("simple" majority) unprofitability is a rule.

EXAMPLE $4(q=3)$. In this case

$$
\begin{aligned}
\mathrm{d}_{Q} & =\frac{\mu(\mu+1)(\mu+2)-(\mu-1)(\mu-2)(\mu-3)}{n(n-1)(n-2)} \\
& =\frac{9 \mu^{2}-9 \mu+6}{n(n-1)(n-2)}=\frac{q}{n} \cdot \frac{3 \mu^{2}-3 \mu+2}{n^{2}-3 n+2},
\end{aligned}
$$

so for majorities which are small enough (satisfy $\mu^{2}-\mu<n^{2} / 3-n$ ) threeperson "agreements" are unprofitable.

EXAMPLE 5 (Simple majority voting games: $\mu=[n / 2]+1$ ). This is the case when the minimal physical majority suffices to win. When $n$ is odd, we have $n=2 \mu-1$ and

$$
\mathrm{d}_{Q}(n, \mu, q)=\frac{U_{\mu-1}^{\mu+q-1}-U_{\mu-q-1}^{\mu-1}}{U_{2 \mu-1-q}^{2 \mu-1}}
$$

Set

$$
r_{1}(\mu, q)=\frac{U_{\mu-1}^{\mu+q-1}}{U_{2 \mu-1-q}^{2 \mu-1}}, \quad r_{2}(\mu, q)=\frac{U_{\mu-q-1}^{\mu-1}}{U_{2 \mu-1-q}^{2 \mu-1}} .
$$

When $\mu / 2 \leq q<n-\mu$, we have the inequality

$$
r_{1}(\mu, q) \leq \frac{\mu(\mu+1)(\mu+2)}{(n-2)(n-1) n}<\frac{q}{n},
$$

so obviously $d_{Q}<q / n$. When $3 \leq q<\mu / 2$, we shall show that the inequality $d_{Q}<q / n$ implies $d_{Q \cup j}<(q+1) / n$ for $j \notin Q$. We have

$$
\begin{aligned}
\mathrm{d}_{Q \cup j}-\mathrm{d}_{Q} & =\left(r_{1}(\mu, q+1)-r_{1}(\mu, q)\right)+\left(r_{2}(\mu, q)-r_{2}(\mu, q+1)\right) \\
& =\frac{1}{n-q}\left[(2 q+1-\mu) r_{1}(\mu, q)+\mu r_{2}(\mu, q)\right] \\
& =\frac{1}{n-q}\left[(2 q+1-\mu) \mathrm{d}_{Q}+(2 q+1) r_{2}(\mu, q)\right]<\frac{2 q+1}{n-q} r_{2}(\mu, q) .
\end{aligned}
$$


Therefore, whenever $r_{2}(\mu, q)<(n-q) /((2 q+1) n)$ - which holds for all $\mu>6$ and $q$ such that $3 \leq q \leq \mu / 2$-we obtain $\mathrm{d}_{q+1}-\mathrm{d}_{q}<1 / n$, which proves the required implication. On the other hand, we know from Example 4 that for $q=3$ and $\mu>6$ (i.e., for odd $n>11$ ) the inequality $\mathrm{d}_{Q}<3 / n$ holds. This implies that for odd $n>11$ and $\mu=(n+1) / 2$ we have $\mathrm{d}_{Q}<q / n$ for every $q \neq 2$ and $q \neq n-\mu$ in the interior case, that is, for every $q$ in the interval $[3, \mu-2]$. (Direct calculations show that the reverse inequality holds for every $q$ in the interior case when $\mu \leq 6$, i.e., $n \leq 11$.)

Similar estimations for $n$ even $(n=2 \mu-2)$ yield

$$
\begin{aligned}
& \mathrm{d}_{Q}<q / n \quad \text { for every } n \geq 20,3 \leq q<n / 2-2 \\
& \text { and every } n \geq 16,4 \leq q<n / 2-2 \text {, } \\
& \mathrm{d}_{Q}=q / n \quad \text { for } n=18, q=3 \text {, } \\
& \mathrm{d}_{Q}>q / n \quad \text { for every } n \leq 14,2 \leq q<n / 2-1 \\
& \text { and for } n=16, q=3 \text {. }
\end{aligned}
$$

The games of simple majority considered in the above example form an extreme case of unprofitability: when the majority required to win increases, the values of $\mathrm{d}_{Q}$ for fixed $q$ also increase, eventually making the operator profitable. This is stated in the following lemma.

LEMma 2. In the interior case, $d_{Q}(n, \mu, q)$ is an increasing function of $\mu$.

Proof. We have

$$
\begin{aligned}
\mathrm{d}_{Q}(n, \mu+1, q)-\mathrm{d}_{Q}(n, \mu, q) & =\frac{U_{\mu}^{\mu+q}-U_{\mu-q}^{\mu}-U_{\mu-1}^{\mu+q-1}+U_{\mu-q-1}^{\mu-1}}{U_{n-q}^{n}} \\
& =\frac{q}{U_{n-q}^{n}} \cdot\left(U_{\mu}^{\mu+q-1}-U_{\mu-q}^{\mu-1}\right)>0 .
\end{aligned}
$$

This lemma together with Lemma 1 and Example 5 implies the following

COROLlaRY. When the number of players is less than 13 or equal to 14, all distrust operators are either neutral (in unanimity games) or profitable (in all other games).

5. Limit behaviour. For games with ten or more players, comparison of $\mathrm{d}_{Q}$ to $q / n$ in the interior case is generally quite cumbersome. However, something can be said about the limit behaviour of the ratio of these two quantities when $n$ tends to infinity and the winning majority percentage, $\mu / n$, has a limit. We consider two cases: $q$ fixed and $q / n$ having a positive limit.

Denote by $\Pi_{Q}(n, \mu, q)$ the ratio of the sums of the Shapley values of all players in $Q$ after and before applying the operator $D_{Q}$ : 


$$
\Pi_{Q}(n, \mu, q)=\frac{\mathrm{d}_{Q}(n, \mu, q)}{q / n}=\frac{n \mathrm{~d}_{Q}(n, \mu, q)}{q} ;
$$

$D_{Q}$ is profitable (resp. unprofitable) for players in $Q$ if and only if $\Pi_{Q}>1$ (resp. $\Pi_{Q}<1$ ).

Proposition 1. Fix $q \geq 2$ and let $\mu_{n}$ be a sequence of integers such that $\mu_{n}>n / 2$ for every $n$ and $\lim _{n \rightarrow \infty} \mu_{n} / n=c$. Then

$$
\lim _{n \rightarrow \infty} \Pi_{Q}\left(n, \mu_{n}, q\right)=q c^{q-1} .
$$

Proof. It is clear that under these assumptions on $\mu_{n}$ and $q$ for almost all $n$ the games $d_{n}^{\mu_{n}}$ are interior. Since

$$
\begin{aligned}
\mathrm{d}_{Q}= & \frac{U_{\mu-1}^{\mu+q-1}-U_{\mu-q-1}^{\mu-1}}{U_{n-q}^{n}} \\
= & \frac{(n-q) !}{n !} \cdot\left[\mu^{q}+(1+\ldots+(q-1)) \mu^{q-1}+K^{+}(\mu)\right. \\
& \left.-\mu^{q}+(1+\ldots+q) \mu^{q-1}+K^{-}(\mu)\right] \\
= & \frac{(n-q) !}{n !} \cdot\left(q^{2} \mu^{q-1}+K(\mu)\right),
\end{aligned}
$$

where $K^{+}, K^{-}$and $K$ are polynomials of degree $q-2$, we obtain

$$
\begin{aligned}
\Pi_{Q}\left(n, \mu_{n}, q\right) & =\frac{n}{q} \cdot \mathrm{d}_{Q}\left(n, \mu_{n}, q\right)=\frac{(n-q) !}{(n-1) !} \cdot\left(q \mu_{n}^{q-1}+q^{-1} K\left(\mu_{n}\right)\right) \\
& =\frac{q \mu_{n}^{q-1}+L\left(\mu_{n}\right)}{n^{q-1}+M(n)}
\end{aligned}
$$

where again $L$ and $M$ are polynomials of degree $q-2$. Therefore, as $n \rightarrow \infty$ and $\mu_{n} / n \rightarrow c, \Pi_{Q}\left(n, \mu_{n}, q\right)$ converges to $q c^{q-1}$.

This result enables us to draw some conclusions about the profitability of distrust operators in games with sufficiently many players. For instance, when $q=3$ and $n \rightarrow \infty, D_{Q}$ will eventually become profitable if $3 c^{2}>1$, i.e., when $\mu \sqrt{3}>n$. With increasing $q$, the threshold ratio $c$ increases to unity.

Proposition 2. Let $q_{n}$ and $\mu_{n}$ be sequences of integers such that $\mu_{n}>n / 2, \lim _{n \rightarrow \infty} q_{n} / n=r, \lim _{n \rightarrow \infty} \mu_{n} / n=c$ and $r<c<1-r$. Then

$$
\lim _{n \rightarrow \infty} \Pi_{Q}\left(n, \mu_{n}, q_{n}\right)=0 \text {. }
$$

Proof. Again, the assumptions ensure that almost all games considered are interior. Using the formula for $d_{Q}$ in the interior case, we obtain

$$
\Pi_{Q}\left(n, \mu_{n}, q_{n}\right)<\frac{n}{q_{n}} \cdot \frac{\mu_{n}\left(\mu_{n}+1\right) \ldots\left(\mu_{n}+q_{n}\right)}{\left(n-q_{n}+1\right) \ldots(n-1) n}<\frac{n}{q_{n}} \cdot\left(\frac{\mu_{n}+q_{n}}{n}\right)^{q_{n}} ;
$$

the quotient in parentheses converges to $r+c$, so is bounded away from unity, and since the exponent $q_{n} \rightarrow \infty$, obviously the limit is zero. 
This proposition also holds for games modified by more than one distrust operator, i.e., games in which a number of pairwise disjoint groups of players have concluded respective agreements. The formulae for values of such games are very complex even for small $n$ because a wide spectrum of cases need to be analysed. However, the generalisation of Proposition 2 to such games is easy even without knowing the formula for the Shapley value: it suffices to observe that if for a given permutation $\pi$ of $N$, the player $\delta(\pi)$ belongs to $Q$, then all players from $Q$ must belong to $\pi^{-1}\left(1,2, \ldots, \mu_{n}\right)$. With $\# Q \rightarrow \infty$ and $\mu_{n} / n \rightarrow c<1$, the probability of this event converges to zero.

Both Proposition 2 and its generalisation, while simple, are of some interest and may even be considered surprising because they sharply contrast with the result of Milnor and Shapley [4]. Milnor and Shapley study games with a fixed number of "large" players holding constant proportions of all votes and an increasing number of "small" players whose individual proportions of votes converge uniformly to zero. A large player in such a game corresponds to a group of players entering a representation ("proxy") agreement as defined by Haller [1], and so the limit theorem by Milnor and Shapley is a counterpart to the generalised version of Proposition 2. But the results obtained are entirely different: in the games studied in [4] the limits of Shapley values of "large" players are positive, and in the interior case representation agreements are in fact always profitable in the limit.

\section{References}

[1] H. Haller, Collusion properties of values, Internat. J. Game Theory 23 (1994), 261281.

[2] M. Malawski, Equal treatment, symmetry and Banzhaf value axiomatizations, ibid., to appear.

[3] - , The Shapley value of majority voting games with multilateral collusion agreements, Bull. Polish Acad. Sci. Math. 42 (1994), 37-42.

[4] J. W. Milnor and L. S. Shapley, Values of large games II: Oceanic games, Math. Oper. Res. 3 (1978), 290-307.

[5] G. Owen, Game Theory, 3rd ed., Academic Press, 1995.

[6] L. S. Shapley, A value for n-person games, in: Contributions to the Theory of Games, Vol. 2, H. Kuhn \& A. W. Tucker (eds.), Princeton Univ. Press, 1953, 307-317.

Institute of Computer Science

Ordona 21

01-237 Warszawa, Poland

E-mail: malawski@ipipan.waw.pl

Received on 30.4.2001;

revised version on 23.1.2002 